\title{
Neural Network based Plant Identification using Leaf Characteristics Fusion
}

\author{
C.S.Sumathi \\ Assistant Professor, \\ School of Post Graduate Studies, \\ Tamil Nadu Agricultural University, \\ Coimbatore - 641003, Tamil Nadu, India
}

\author{
A.V.Senthil Kumar \\ Director, Department of Post Graduate and \\ Research in Computer Applications, \\ Hindusthan College of Arts and Science, \\ Coimbatore - 641028, Tamil Nadu, India
}

\begin{abstract}
A computerized method for recognizing plant leaf based on their images is proposed. Plant classification is based on leaf identification which has broad application on prospective in medicine and agriculture. Plant leaf images corresponding to six plant types are taken using a digital camera which are examined using three different modeling techniques, first based on Multi Layer Perceptron (MLP) Neural network and second on Normalized Cubic Spline Feed Forward Neural network (NCS-FNN) and third on proposed NCS-FNN for real data. Correlation based feature selection (CFS) is considered to produce a ranked list of attributes. Matlab is used to extract the leaf features such as edge and texture. Edge and texture are the important visual attribute which can be used to describe the pixel organization in an image. Further to increase the accuracy in NCS-FNN the neural network is trained using a back propagation rule by back propagating errors and changing weights of node. The dataset consists of 197 images which are divided into six classes.
\end{abstract}

\section{Keywords}

Leaf Identification, Leaf Features, Fusion, Correlation Feature Selection, Mat Lab, Multilayer Perceptron, Normalized Cubic Spline-Feed Forward Neural Network.

\section{INTRODUCTION}

Plants are the source of providing oxygen, food, medicine, fuel, and much more, hence it is considered as the essential part of life on earth [1]. A detailed understanding of plants is essential to increase the agricultural productivity and sustainability. With an unavoidable growing human population and a varying of climate, there is an increasing threat to many ecosystems. Therefore it is vital to identify a new or rare species and to measure their geographical scope as a part of wider biodiversity scheme. So the need of the hour is plant recognition and classification. Compared with methods, such as cell and molecule biology methods, the classification based on leaf image is the foremost choice for classification of leaf plant. Sampling and capturing of leaves is inexpensive but convenient. The captured leaf image can be easily transferred to a computer and necessary features are automatically extracted by using image processing techniques and subsequently it recognizes the leaf.

Leaves are the plants powerhouse and major site of food production for the plant is through leaves. Leaves sizes and shapes can be varied and they are often used to identify plants. But some of the plants having same leaf structure where the identification based on the leaf structure are very difficult. Fruit trees like mango (Mangifera indica) and sapota (Manilkara zapota) has the leaves as pinnate venation with the wavy edges and moreover both leaves are harder. The plants like cotton (Gossypium sp.) and sunflower (Helianthus annuus) have pinnate venation and stem has alternates in it. Because of the same structure, it is very difficult to differentiate both cotton and sunflower plants during vegetative stages even a sunflower leaf is heart-shaped. Hence, Plant leaf images classification using the modeling techniques of proposed NCS-FNN for real data produces a better accuracy with higher percentage.

Data mining is considered as the analysis of large observational data sets to find an unsuspected relationship and to summarize the data in a novel ways that are understandable as well as useful to the data owner. Data mining tools is used for forecasting the future trends and activities in order to support the decision of people [2]. Also this tool is a discipline which incorporates statistics, data management and databases, machine learning, pattern recognition, and artificial intelligence. Often it is set into the broader framework of Knowledge Discovery in Databases (KDD) process. The KDD process includes some stages in it which are as follows: selecting the target data, data pre-processing, transforming the preprocessed data if necessary by performing the data mining to extract patterns and relationships, and also interpreting and assessing the discovered structures.

Classification and association rule mining is considered as the two main tasks of Data Mining. In classification rule mining, mapping of instances into distinct classes is possible. Classification is used for assigning an object to one of several predefined categories. Also it includes predicting the value of a (categorical) attribute (the class) based on values of other attributes (that is predicting attributes). Association rule mining is capable of revealing all the interesting relationships in a large database and these relationships show a strong association between an attribute-value pairs (or items) that frequently occur in a given data set. Set of association rules is used for describing the relationships in databases, and for discriminating between the different kinds or classes of database instances.

\section{NEURAL NETWORK}

This model is a set of computational elements (neurons) are interconnected with each other. The neurons often mapped as: input layer, hidden layer and output layer. The advantage of neural network is that the functional relationship between dependent variable and independent variable does not have to be a pre-specified one. Also network learns the relationship from a sample dependent and independent variables which are used to train the network [3]

\subsection{Related Works}

The geometrical features of lentil seeds (Lens culinaris Medik) by using the image analysis LUCIA system Ver.3.52 [4]. The values of the weight of 1000 kernels, kernel density, specific volume, specific surface area, and surface area of 1000 kernels of red and green lentils were determined as 66.61 and $138.56 \mathrm{~g}$, 
1504.5 and $1376.4 \mathrm{~kg} / \mathrm{m} 3,0.6647$ and $0.7265 \mathrm{~cm} 3 / \mathrm{g}, 0.594$ and $0.579 \mathrm{~m} 2 / \mathrm{kg}, 395.4$ and $801.9 \mathrm{~cm} 2$, respectively. The lentil volume has been simulated using an oblate spheroid and two sphere segments where the volumes obtained with both models were compared with that obtained by psychometric method. Percentage differences of the two sphere segment approximation of red and green lentils were $4.4 \%$ and $4.2 \%$, respectively. The height (thickness) of lentils was constant and practically same with both varieties $(2.6 \mathrm{~mm})$ and it was possible to simplify the geometrical models. As a result, 2D image analysis was suitable for a quick evaluation of the specific volume and surface area of grains on the basis of the projected area (equivalent diameter) without the height measurement.

A study which intends an idle case approach in plant leaf classification and recognition which was applicable in the real world, and also acceptable in the lab [5]. The consequences from this study introduced more external factors for consideration while experimenting a real world sampling for leaf recognition and classification does this. This study uses the types of leaf features that was extracted, external factors that was considered before extraction process, types of extraction and classification methods that was used for leaf recognition and classification. The selected classifiers for the test includes Probalistic Neural Network (PNN) with color and texture, Linear Discriminant Analysis(LDA) with Nearest Neighbor (LNN) and General Regression Neural Network(GRNN).

A texture segmentation algorithm for visual information processing was proposed [6]. The multichannel filtering approach for texture analysis was intuitively appealed because the dominant spatial-frequency components of different textures were different. Texture features was obtained by subjecting each filtered image to a nonlinear transformation and energy in a window around a pixel is calculated. In texture segmentation, neighboring pixels were likely to belong to the same texture category. A method has been proposed that incorporates the spatial adjacency information directly in the clustering process. This was achieved by including the spatial coordinates of the pixels as two additional features. An experimental result on images with natural texture as well as artificial texture with identical 2 nd and 3rd order statistics was reported.

The contour-based approach where plant leaves were analyzed by using their outer boundaries[7], the application of square root velocity function for plant leaf classification was investigated based on the shape of their boundaries and compared with three other methods such as shape distribution (D2), Gaussian Euclidian Distance Transform of the shape boundaries (GEDT) and Multi Scale Distance Matrix (MSDM). Additionally the difference in leaf shapes has been seen as elastic deformations, which captures with a Riemannian elastic metric. Square root velocity function outperforms previous techniques in 19 out of the 32 species, while the D2, GEDT, and MSDS perform the best on three, three, and seven species respectively.

In an automated system for recognizing a plant species based on leaf images[8], plant leaf images were analyzed using two different shape modeling techniques such as based on the Moments-Invariant (M-I) and Centroid - Radii (C-R) model. A hybrid set of features involves both the M-I and C-R models which have been generated and explored to find whether the combination feature vector can lead to better performance. For computing recognition rates, a comparison between training and testing sets has done using MLP with feed-forward backpropagation architectures. Automated discrimination between three leaf shapes was done with variety of approaches to find the optimum results. A salient feature of the current approach was the low-complexity data modeling scheme which was used for dimensionality of the feature vectors which were typically below 40 .

The necessity to use the hand writer identification with use of the feed forward neural networks is justified here [9]. They have presented some practical aspects of the automatic handwriting identification using scanned images with feed forward neural networks. After training of feed forward neural network with samples of three known hand writers it was verified if the feed forward neural network was good trained. With the analyzes of three unknown letters, neural network was analyzed and decided the next results. The result of each found letter processing by Neural network has been stored in a vector for computing a mean. The probability of identifying a writer was given by the above mean. Results for the verity the fundamental dogmas underpinning handwriting identification were: no two people write exactly alike and no one person writes exactly the same way twice.

The MLPNN and its function approximation is examined and described [10]. The back propagation algorithm has the principle procedure for training MLP. When they designed MLP network to analyze the properties of Jordan oil Shale, the following facts were reached; MLP with a lot of hidden layers performs better when compared with one or two hidden layer with regard of the output performance parameters. When compared between the output of MLP and mathematical formula, it was found that their output performance parameters were best suited with the experimental. This obtained result was completely matched with the main concepts of theoretical visions of ANN.

\section{MATERIALS AND METHODS}

\subsection{Feature Extraction}

The process of detecting the pixels in the image that represent the edges of the image object is named as edge detection. This edge detection process consists of three steps such as: filtering, enhancement and detection. Noise in the image removed during filtering due to random variation in intensity value. Enhancement intensifies the pixels while there is a change in local intensity. Edges are detected using thresholding concept. Robert edge detection, Sobel edge detection, Prewitt edge detection and canny edge detection are most commonly used detection methods and Sobel edge detector is proposed here.

The Sobel edge detector finds the approximate absolute gradient magnitude to detect edges at each point. Regions of high spatial frequency corresponding to edge are obtained by the 2-D gradient measurement. A series of gradient magnitudes can be created using a simple convolution kernel and these convolutions can be mathematically represented as:

$N(x, y)=\sum_{k=1}^{1} \sum_{j=1}^{1} K(j, k) p(x-j, y-k)$

The Sobel detector uses two convolution kernels for detecting changes in horizontal contrast $\left(\mathrm{h}_{\mathrm{y}}\right)$ and vertical contrast $\left(\mathrm{h}_{\mathrm{x}}\right)$.

Gabor filters is used extensively in image processing for its ability to perform multi-resolution decomposition. This feature in Gabor filter is available because of its localization in spatial and spatial frequency domain. Also this feature makes Gabor filters ideal for texture segmentation since simultaneous measurements in both spatial and spatial-frequency domains is required. Filters with lower bandwidths are a desirable factor in the spatial-frequency domain as they allow finer distinctions 
making among different textures. To locate texture boundaries filters that are localized in the spatial domain are desired. The relation between the two is inversely related based on the uncertainty principle [11].

A Gabor function is a sinusoidal modulated Gaussian in the spatial domain. For a 2-D Gaussian curve with a spread of $\sigma_{x}$ and $\sigma_{y}$ in the $\mathrm{x}$ and y directions, respectively, and a modulating frequency of $u_{o}$, the real impulse response of the filter [12] is given by

$$
h(x, \mid y)=\frac{1}{2 \pi \sigma_{x} \sigma_{y}} \exp \left\{-\frac{1}{2}\left[\frac{x^{2}}{\sigma_{x}^{2}}+\frac{y^{2}}{\sigma_{y}^{2}}\right]\right\} \cdot \cos \left(2 \pi u_{0} x\right)
$$

\subsection{Feature Selection}

Feature selection is the process of deciding on a subset of relevant features for use in system construction. The central assumption of using a feature selection technique is that the data contains many redundant or irrelevant features in it. Redundant features are those which provide only information about the currently selected features, whereas irrelevant features provide no useful information. Feature selection techniques are a subset of the more general field of feature extraction where feature selection returns a subset of the features. Selection techniques are used in domains where there are many features and comparatively few samples [13].

A feature $\mathrm{V}_{\mathrm{i}}$ is said to be relevant iff there exists some vi and $\mathrm{c}$ for which

$\mathrm{p}(\mathrm{Vi}=\mathrm{vi})>0$ such that

$$
\mathrm{p}\left(\mathrm{C}=\mathrm{c} \mid \mathrm{V}_{\mathrm{i}}=\mathrm{v}_{\mathrm{i}}\right) \neq \mathrm{p}(\mathrm{C}=\mathrm{c}) .
$$

CFS is a filter algorithm which ranks feature subsets based on a correlation based heuristic evaluation function. The bias of the evaluation function is toward subset which includes a feature that is highly correlated with the class and uncorrelated with each other. Empirical evidence from the feature selection literature shows that, along with irrelevant features, redundant information must be eliminated as well $[14 ; 15 ; 16]$. A feature is said to be redundant if one or more of the other features are highly correlated with it. Heuristic search is used to traverse the space of feature subsets in a reasonable time; the subset with the highest weight found during the search is reported.

\subsection{Existing System}

\subsubsection{MLP}

MLP belongs to a feed forward artificial neural network model that maps a set of input data onto a set of its appropriate outputs. MLP includes multiple layers of nodes in a directed graph, and each layer is fully connected to the next one. Except the input nodes, each node is considered as a processing element or a neuron with a nonlinear activation function. MLP generally make use of back propagation for training the network [17]. Learning occurs in the perceptron by changing the connection weights once every piece of data gets processed based on the amount of error occurred in the output when compared to the expected result. This example of supervised learning can be carried out through the back propagation, i.e., a generalization of the least mean squares algorithm in the linear perceptron.

The error in the output node ' $j$ ' in $n^{\text {th }}$ data point is given by

$e_{j}(n)=d_{j}(n)-y_{j}(n)$ where $d$ is the target value and $y$ is the value that are produced by the perceptron.

Corrections to the weight of nodes is based on those corrections which minimize the error in the entire output and is given by

$E(n)=-\frac{1}{2} \sum_{j} e_{j}^{2}(n)$

\subsubsection{NCS-FFNN}

Neural networks are made up of multiple layers of neurons or computational units where all the neurons are interconnected with each other. The inputs are fed on the input layer which propagates through the network in forward direction through the hidden layers to give an output. Output signal can be calculated using weights, bias and activation function. The neural network is trained by using back propagation rule by back propagating the errors and changing weights of nodes. The error is the difference between the outputs obtained and desired output. The following are the algorithms which can be used for calculating various parameters involved in training a neural network

The total input for a given neuron is given as:

$S_{k}=\sum w_{j k} y_{k}+\theta_{k}$

where $S_{k}$ is the total or effective input for unit $\mathrm{k}, w_{j k}$ the weight of the connection, $y_{k}$ is current activation and $\theta_{k}$ is the bias.

Activation function $A_{f}$ takes the input and current activation and gets the new activation value during learning by:

$y_{k}(t)=A_{f}\left(y_{k}(t-1) \cdot s_{k}(t-1)\right)$

The value of activation functions are generally limited to 0,1 using a threshold function. Most commonly used is sigmoid function.

$y_{k}=A\left(s_{k}\right)=\frac{1}{1+e^{-s k}}$

The spline-based NN is built with generalized sigmoidal (GS) neuron, which consists of an adaptive parametric spline activation function [18] where the spline activation is easy to adapt and implement. It also retains a squashing property of the sigmoid and smoothing characteristics. MLP can built using spline activation function are universal approximates and have smaller structural complexity.

The spline activation function reproduces the shape of whole cubic spline along with the directions specified by wj, $\mathrm{j}=1, \ldots, \mathrm{n}$ [19].

$\varphi\left(w_{j} x\right) \sum c_{i}\left|w_{j} x-\alpha_{i j}\right|^{3}$

$f(x)$ can be written as:

$f(x)=\sum_{j=1}^{n} \mu_{j} \varphi_{j}\left(w_{j} x\right)$ 
$\mu_{j}$ and $w_{j}$ are found by using back propagation, thus optimal set of parameters and coordinates are found. The tracts in the spline are described by combination of coefficients. Local spline basis functions are controlled by only 4 coefficients are used to represent the activation function. Catmull-Rom cubic spline is used and its $\mathrm{i}^{\text {th }}$ tract is expressed as:

$$
F_{i}(u)=\frac{\left[F_{x, i}(u)\right]}{\left.--F_{y, i}(u)\right]}=\frac{1}{2}\left[u^{3} u^{2} \text { u } 1\right]
$$

\section{RESULTS AND DISCUSSION}

Six species of 197 leaves of similar structures viz., mango, sappota, guava, neem, cotton, are used. Sample images are shown figure 1 .
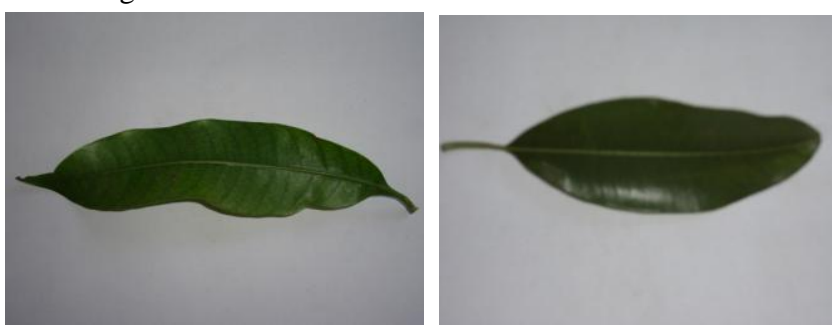

Figure 1: Leaves sample used

The features like edge and texture is extracted using Matlab. The features extracted are used to train the classification algorithms. The features are classified using MLP, NCS-FFNN. The classification accuracy obtained is given in Table 1 and Figure 2. Table 2 tabulates the precision, recall and f Measure for various algorithms and compared with the proposed method. Figure 3 to 5 shows the result graph of precision, fMeasure and recall and compared with MLP and proposed techniques such as Proposed NCSFFNN and Proposed NCSFFNN for real data.

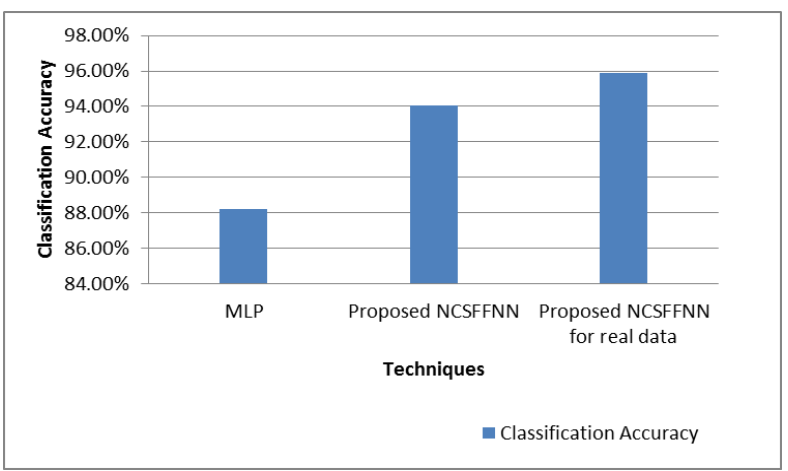

Figure 2: Classification Accuracy

From table 1 and figure 2 it is observed that the Classification Accuracy is calculated for different techniques. The Proposed NCSFFNN has $6.67 \%$ of better classification accuracy than MLP and Proposed NCSFFNN for real data has $8.72 \%$ and $1.92 \%$ of better classification accuracy than MLP and Proposed NCSFFNN respectively.

Table 1. Classification Accuracy

\begin{tabular}{|l|c|}
\hline Technique Used & Classification Accuracy \\
\hline MLP & $88.20 \%$ \\
\hline Proposed NCSFFNN & $94.08 \%$ \\
\hline Proposed NCSFFNN for real data & $95.89 \%$ \\
\hline
\end{tabular}

Table 2. Precision, Recall and F Measure

\begin{tabular}{|l|c|c|c|}
\hline Technique Used & Precision & Recall & f Measure \\
\hline MLP NCS & 0.88 & 0.88 & 0.88 \\
\hline $\begin{array}{l}\text { Proposed fFNN } \\
\text { FFr }\end{array}$ & 0.96 & 0.94 & 0.91 \\
\hline $\begin{array}{l}\text { Proposed } \\
\text { NCSFFNN for } \\
\text { real data }\end{array}$ & 0.96 & 0.95 \\
\hline
\end{tabular}

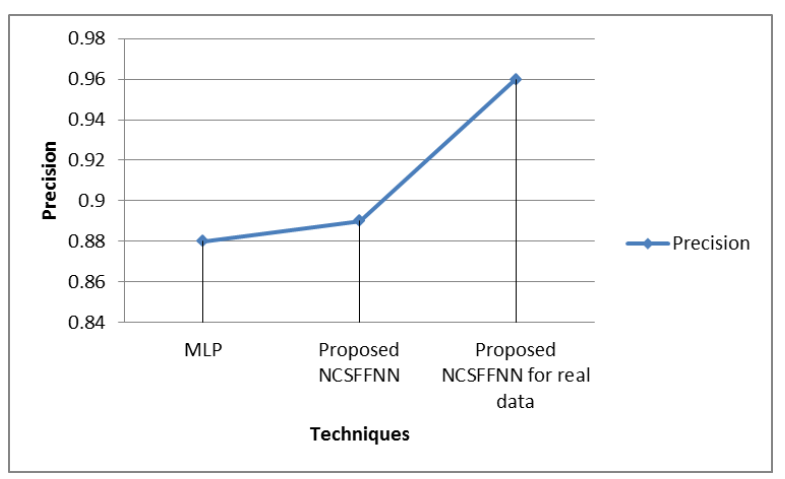

Figure 3: Precision

From table 2 and figure 3 it is observed that the Precision is calculated for different techniques. The Proposed NCSFFNN has $1.14 \%$ of better Precision than MLP and Proposed NCSFFNN for real data has $9.09 \%$ and $7.87 \%$ of better Precision than MLP and Proposed NCSFFNN respectively.

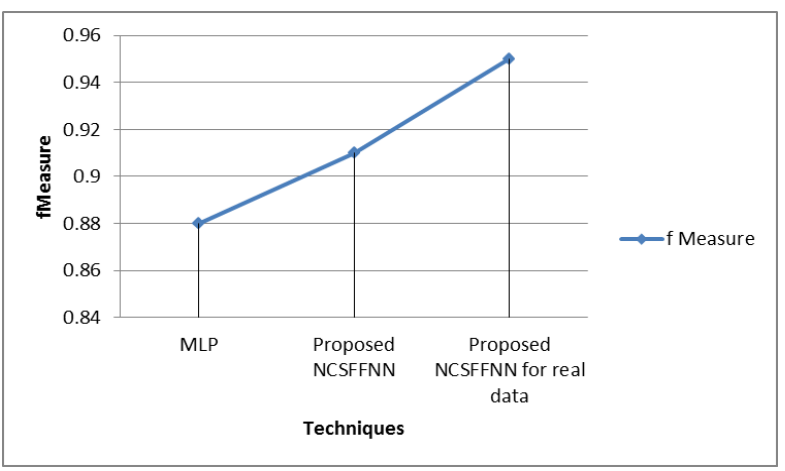

Figure 4: f Measure

From table 2 and figure 4 it is observed that the fMeasure is calculated for different techniques. The Proposed NCSFFNN has $3.41 \%$ of better fMeasure than MLP and Proposed NCSFFNN for real data has $7.95 \%$ and $4.4 \%$ of better fMeasure than MLP and Proposed NCSFFNN respectively.

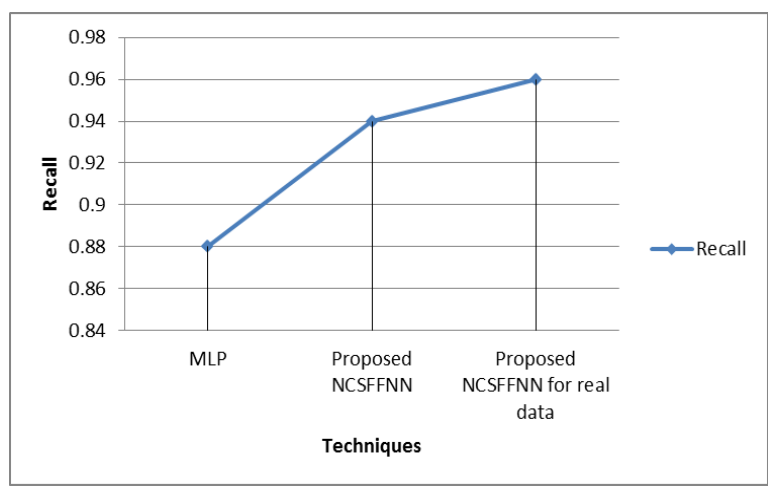




\section{Figure 5: Recall}

From table 2 and figure 5 it is observed that the Recall is calculated for different techniques. The Proposed NCSFFNN has $6.82 \%$ of better Recall than MLP and Proposed NCSFFNN for real data has decrease in Recall $3.19 \%$ and $3.41 \%$ than MLP and Proposed NCSFFNN respectively.

\section{CONCLUSION}

The Gabor filter and sobel edge detector is used as the feature extraction. Feature selection is applied in this paper. The existing MLP and how the proposed method is applied is explained. Matlab is used here to extract the leaf features such as edge and texture where edge and texture are the visual attribute which can be used to describe the pixel organization in an image. From the experimental results it is observed that the Proposed NCSFFNN has $6.67 \%$ of better classification accuracy than MLP and Proposed NCSFFNN for real data has $8.72 \%$ and $1.92 \%$ of better classification accuracy than MLP and Proposed NCSFFNN respectively.

\section{REFERENCES}

[1] Kulkarni, A.H., Rai, H.M., Jahagidar, K.A. and Upparamani, P.S.2013. A Leaf Recognition Technique for Plant Classification Using RBPNN and Zernike Moments. International Journal of Advanced Research in Computer and Communication Engineering, 2(1): 984-988

[2] Prachitee, Shekhawat, Sheetal, S. and Dhande. 2011 Building an Iris Plant Data Classifier Using Neural Network Associative Classification. International Journal of Advancements in Technology, Vol 2, No 4, 491-506. ISSN0 976- 4860

[3] Jamal, A. M. M. and Sundar, C. 2011. Modeling exchange rates with neural networks. Journal of Applied Business Research (JABR), 14(1): 1-6.

[4] Firatligil-Durmuş E., Šarka, E. and Bubnik, Z. 2008. Image vision technology for the characterization of shape and geometrical properties of two varieties of lentil grown in Turkey. Czech J. Food Sci., 26:109-116.

[5] Ab Jahal, M. F., Suhurdi Hamid, Salehuddin Shulb and Illiasaak Ahmed, 2013. Leaf Features Extractions and Recognition Approaches to Classify Plant. Journal of Computer Science 9(10): 1295-1304.

[6] Jain, A. K., Ratha, N. K. and Lakshmanan, S. 1997. Object detection using Gabor filters. Pattern Recognition, 30(2): 295-309.

[7] Laga, H., Sebastian Krutek, Anuj Srivastava, Mahmood Golzarian and Stanley Miklavcic. 2012. A Riemannian Elastic Metric for Shape-Based Plant Leaf Classification. Proceedings of the International Conference on Digital Image Computing Techniques and Applications (DICTA), 3-5 Dec. Western Australia.
[8] Chaki, J. and Ranjan Parekh. 2011. Plant Leaf Recognition using Shape based Features and Neural Network Classifiers. International Journal of Advanced Computer Science and Application, 2(10): Vol. 2, No. 10, 2011

[9] Constantin Anton, Cosmin Stirbu and Romeo-Vasile Bades. 2010. Identify Handwriting individually Using Feed Forward Neural Networks. International Journal of Intelligent Computing Research, 1(4) : Page No. 183-188

[10]Jamal M., Nazzal, M., Ibrahim, El-Emary and Salam A. Najim. 2008. Multilayer Perceptron Neural Network (MLPs) for Analyzing the Properties of Jordan Oil Shale. World Applied sciences journal, 15(5):546-522. ISSN 1818-4952.

[11] Samad, R. and Sawada, H. 2011. Edge-based Facial Feature Extraction Using Gabor Wavelet and Convolution Filters. MVA, June 13-15, 2011, 430-433.

[12] Sumathi, C. S. and Senthil Kumar, A. 2012. Edge and Texture Fusion for Plant Leaf Classification. International Journal of Computer Science and Telecommunications, 3(6): 6-9.

[13] Mark A. Hall. 1999. Correlation-based Feature Selection for Machine Learning, 51-74

[14] Langley, P. and Sage, S. 1994. Induction of selective Bayesian classifiers. Proceedings of the Tenth conference on Uncertainty in Artificial Intelligence, Jul 29-31 1994, Seattle, W.A, Morgan Kaufmann, 399-406

[15] Kohavi, R. and John, G. 1996. Wrappers for feature subset selection. Artificial Intelligence, special issue on relevance. 97(1-2):273-324.

[16] Kohavi, R. and Sommerfield, D. 1995. Feature subset selection using the wrapper method: Overfitting and dynamic search space topology. Proceedings of the First International Conference on Knowledge Discovery and Data Mining. AAAI Press.

[17]Han, J. and Kamber, M. 200. Data Mining Concepts and Techniques, Han, J. and Kamber, M. 2002. Data Mining Concepts and Techniques, Morgan Kaufmann USA, 155860-489-8.

[18]Guarnieri, S., Piazza, F. and Uncini, A. 1995. Multilayer neural networks with adaptive spline-based activation functions. Proceedings of the International Neural Network Society Annual Meet, WCNN, Washington, DC, pp. I695- I699.

[19] Vecci, L., Piazza, F. and Uncini, A. 1998. Learning and approximation capabilities of adaptive spline activation neural networks. Neural Networks,11(2): 259-270. 